\title{
Malaria epidemiology and interventions in Ethiopia from 2001 to 2016
}

\author{
Hiwot S Taffese ${ }^{1}$, Elizabeth Hemming-Schroeder ${ }^{2}$, Cristian Koepfli ${ }^{2}$, Gezahegn Tesfaye ${ }^{1}$, Ming-chieh Lee ${ }^{2}$, \\ James Kazura ${ }^{3}$, Gui-Yun Yan ${ }^{2}$ and Guo-Fa Zhou ${ }^{2 *}$
}

\begin{abstract}
Background: Ethiopia is one of the African countries where Plasmodium falciparum and P. vivax co-exist. Monitoring and evaluation of current malaria transmission status is an important component of malaria control as it is a measure of the success of ongoing interventions and guides the planning of future control and elimination efforts.

Main text: We evaluated changes in malaria control policy in Ethiopia, and reviewed dynamics of country-wide confirmed and clinical malaria cases by Plasmodium species and reported deaths for all ages and less than five years from 2001 to 2016. Districts level annual parasite incidence was analysed to characterize the malaria transmission stratification as implemented by the Ministry of Health. We found that Ethiopia has experienced major changes from 2003 to 2005 and subsequent adjustment in malaria diagnosis, treatment and vector control policy. Malaria interventions have been intensified represented by the increased insecticide treated net (ITN) and indoor residual spraying (IRS) coverage, improved health services and improved malaria diagnosis. However, countrywide ITN and IRS coverages were low, with 64\% ITN coverage in 2016 and IRS coverage of 92.5\% in 2016 and only implemented in epidemic-prone areas of $>2500$ m elevation. Clinical malaria incidence rate dropped from an average of 43.1 cases per 1000 population annually between 2001 and 2010 to 29.0 cases per 1000 population annually between 2011 and 2016. Malaria deaths decreased from 2.1 deaths per 100000 people annually between 2001 and 2010 to 1.1 deaths per 100000 people annually between 2011 to 2016. There was shrinkage in the malaria transmission map and high transmission is limited mainly to the western international border area. Proportion of $P$. falciparum malaria remained nearly unchanged from 2000 to 2016 indicating further efforts are needed to suppress transmission.
\end{abstract}

Conclusions: Malaria morbidity and mortality have been significantly reduced in Ethiopia since 2001, however, malaria case incidence is still high, and there were major gaps between ITN ownership and compliance in malarious areas. Additional efforts are needed to target the high transmission area of western Ethiopia to sustain the achievements made to date.

Keywords: Malaria control, Policy, Ethiopia, Plasmodium falciparum, Plasmodium vivax, Epidemiology, Incidence, Spatial distribution

\section{Multilingual abstracts}

Please see Additional file 1 for translation of the abstract into the five official working languages of the United Nations.

\section{Background}

Malaria morbidity and mortality have been significantly decreased in Ethiopia and worldwide in the past decade

\footnotetext{
* Correspondence: zhoug@uci.edu

${ }^{2}$ Program in Public Health, University of California, Irvine, CA 92697, USA

Full list of author information is available at the end of the article
}

[1]. Ethiopia's fight against malaria started many years ago and transmission of this infectious disease significantly decreased since 1959 [1, 2]. However, malaria still remains a major public health problem in Ethiopia [1]. Ethiopia has a population of nearly 100 million, and it is estimated that $\sim 68 \%$ of the population is at risk of the disease [1, 3]. Plasmodium falciparum and $P$. vivax co-exist as major parasite species in Ethiopia [1]. This epidemiologic feature makes malaria control more complicated than in most African countries where P. vivax has low or nil endemicity.

(C) The Author(s). 2018 Open Access This article is distributed under the terms of the Creative Commons Attribution 4.0 International License (http://creativecommons.org/licenses/by/4.0/), which permits unrestricted use, distribution, and 
Malaria transmission in Ethiopia occurs mainly at altitudes $<2000 \mathrm{~m}$, although endemic regions $>2000 \mathrm{~m}$ have been reported [3-5]. The levels of malaria risk and transmission intensity, however, show marked seasonal, inter-annual and spatial variability, with the exception of the southwestern international border low land area where transmission is year-around [6-8]. In most regions of the country, the major transmission season is from September to December, following the main rainy season from June to September [3]. There is a short transmission season from April to May following the short rainy season in some regions [3]. Anopheles arabiensis is the predominant vector with An. pharoensis, An. coustani, An. funestus and An. nili having a minor role in transmission [9]. Generally, the diverse ecology of the country supports a wide range of transmission intensities ranging from low-seasonal to high-perennial transmission. For planning purposes and targeting of intervention strategies, the Federal Ministry of Health $(\mathrm{FMoH})$ of Ethiopia has stratified the country's malaria transmission burden using 'woreda' (district)-level transmission intensity according to annual parasite incidence per 1000 population (API) and elevation [3, 10]. Accordingly, four broad strata were identified by the mixed criteria of the $\mathrm{FMoH}$ and World Health Organization (WHO) - malaria free, low, moderate, and high transmission $[3,9]$.

Plasmodium falciparum are endemic in many regions of the country $[3,11-13]$. Plasmodium malariae and $P$. ovale infection are uncommon and account for $<1 \%$ of confirmed malaria cases $[3,11]$. Chloroquine $(C Q)$ is currently the recommended first-line drug for treatment of vivax malaria [11]. In vivo monitoring of uncomplicated vivax malaria cases indicates that the CQ is generally efficacious; however, treatment failures have been reported [14-17]. The success of malaria control efforts has largely depended on financial support from donor funds [1].

In the past decade Ethiopia has made significant strides in expanding coverage of key malaria interventions throughout the country. Indoor residual spraying (IRS) using dichlorodiphenyltrichloroethane (DDT) was introduced in 1959 with the global malaria eradication campaign, and since then different chemical insecticides have been used for malaria control $[9,18,19]$. Insecticide-treated nets (ITN) were introduced in 1997 as an additional intervention [10]. Chloroquine was the first line treatment of all malaria species in Ethiopia before 1998. It was replaced by sulfadoxine-pyrimethamine (SP) after 1998 for the treatment of uncomplicated $P$. falciparum due to widespread decline in the efficacy of CQ [20,21]. Parasites soon developed resistance to SP drugs [22, 23]. Planning for scaling-up malaria prevention and control interventions started in 2003 with the support from the Global Fund to Fight AIDS, Tuberculosis and Malaria (GFATM) [3]. In 2004, the FMoH introduced artemisinin-based combination therapy (ACT) as the first-line drug for treatment of $P$. falciparum malaria as well as rapid diagnostic tests (RDT) to improve diagnosis and long-lasting insecticidal nets (LLINs) as a method of preventing transmission of parasite from mosquitoes to people [3]. Major scale-up began in 2005 with country-wide distribution of RDTs, ACTs, LLINs and implementation of IRS [3].

The aim of this study was to provide comprehensive evaluation of changes in national malaria control policy and progress made in malaria control interventions and malaria epidemiology in Ethiopia. We discuss obstacles to malaria elimination and review national malaria data from 2000 to 2016 and analyse these data in order to advance understanding of how changes in policy have impacted temporal changes in malaria transmission in the country.

\section{Main text \\ Data collection}

Changes in malaria control policy were reviewed based on different sources including $\mathrm{FMoH}$ publications on malaria diagnosis, treatment and vector control guidelines, WHO guidelines, the President's Malaria Initiative (PMI), and Ethiopian malaria control operational plans and reports [10, 11, 18, 19, 24-30]. National malaria data from health facilities were collected through the Health Management Information System (HMIS). Total confirmed and clinical malaria cases, malaria cases by species, and reported deaths for all ages and age $<5$ years between 2001 and 2016 were reviewed. Incidence rate was expressed as malaria cases/1000 people/year and death rate as deaths/100000 people/year. Changes in number of health facilities, annual number of tests (microscopic examination of blood smears and RDT) of suspected malaria cases, reports on new insecticide treated nets (ITN and LLIN), and IRS coverage from 2007 to 2016 were obtained from the $\mathrm{FMoH}$. The interventions were targeted to suit local epidemiological situations, with case management being strengthened in all areas. LLIN and IRS were implemented in targeted areas before 2011 [3].

Annual parasite incidence (API) according to district was analysed to characterize the district as malaria-free (API < 1.0), low (1.1-5.0), moderate $(5.1-100)$, or high transmission $(>100)$ and to map transmission dynamics according to district [10]. Malaria records at the district level nation-wide were only available since 2013, and annual district level micro-plans were used to determine API of each district in 2013 and 2016 (with the exception of the Somali region where information from the National Public Health Emergency Management Plan was used). Data from a total of 845 districts were analysed. 


\section{Statistical analysis}

API and malaria death rates were calculated based on 2007 Ethiopian government census populations and 2013 projections at district and national levels assuming constant population growth rate over the study period. Percent changes in malaria indicators from 2013 to 2016 were calculated according to the formula: (Value2013Value2016)/Value2013 $\times 100$. Maps of API at district level were generated using ArcGIS 10.0 (ESRI, Redlands, CA, USA). Numbers of districts with malaria-free, low, moderate and high API were calculated separately for 2013 and 2016. The total population and percentage composition in different transmission categories was calculated accordingly. The number of districts and total populations in different transmission categories were compared between 2013 and 2016 using $X^{2}$-test through free online statistical tool VassarStats: Website for Statistical Computation (http://vassarstats.net/ index.html).

\section{Evolving malaria control policy}

The major policy changes in treatment were the replacement of CQ with SP for the first line treatment of uncomplicated P. falciparum malaria in 1998 and subsequent replacement with artemether-lumefantrine (AL), an ACT drug, in 2004 (Fig. 1). Also in 2004, RDTs were implemented in Ethiopia to improve diagnosis; however, the test-treat policy, i.e., administration of antimalarial drugs was based on test results, was implemented in 2010 (Fig. 1). According to current national guidelines, laboratory diagnosis is based on microscopic examination of blood smears at hospitals and health centres. RDTs are used at health posts and by health extension workers based in local communities.

CQ remains the first-line treatment of uncomplicated vivax malaria (Fig. 1) [14-17]. Anti-relapse therapy with primaquine for $P$. vivax malaria is currently recommended, with supervised use, in malaria elimination-designated districts [11].
LLIN were introduced into Ethiopia in 2004 as a method of malaria prevention and control [3]. Major scale-up began in 2005 with country-wide distribution of LLINs free of charge (Fig. 1) [3]. LLIN distribution campaigns were carried out in 2005, 2010 and 2015. Before 2011 LLIN coverage targeted all age groups in endemic areas below $2000 \mathrm{~m}$ altitude. Long lasting insecticidal nets have been provided free in all malaria endemic areas since 2011 [27-29].

DDT and malathion were the main insecticides used for IRS before 2007 (Fig. 1) [9]. Due to concerns of high-level resistance to and long-term field residual effect of DDT, pyrethriods were used for IRS from 2007 to 2010. Bendiocarb and propoxur were used in different districts in 2011 and 2012, respectively. Pirmiphos-methyl was introduced in PMI spraying districts in 2016 (Fig. 1) [9, 31]. Indoor residual spraying was used in epidemic-prone areas situated at altitudes of 2000 to $2500 \mathrm{~m}$. [3]. New guidelines promote selectively IRS intervention based on API, i.e., IRS is used in both low (API 1 5) and high transmission areas (API $\geq 100$ ). IRS is also used in low-risk populations located in highland epidemic fringe areas [25, 28], however, this new recommendation has not been implemented in the field at the time this study was conducted.

Larval source reduction and laviciding has been recommended as part of the vector control measures [11, 32]. Larval control is recommended for all malaria affected areas where breeding sites are permanent, identifiable, and few $[25,30]$. Temephos $\left(\mathrm{Abate}^{\circ}\right)$ is the recommended larvicide [11].

\section{Changes in clinical malaria incidence}

There has been an overall reduction in reported malaria incidence and deaths, increases LLIN and IRS coverage and the number of health facilities from 2000 to 2016 (Fig. 2 and Table 1). Population pooled microscopically or RDT confirmed cases increased from $<20 \%$ before 2008 to $\sim 40 \%$ by 2012 and $>80 \%$ since 2013 (Fig. 2). There were three small malaria case increases in 2004,

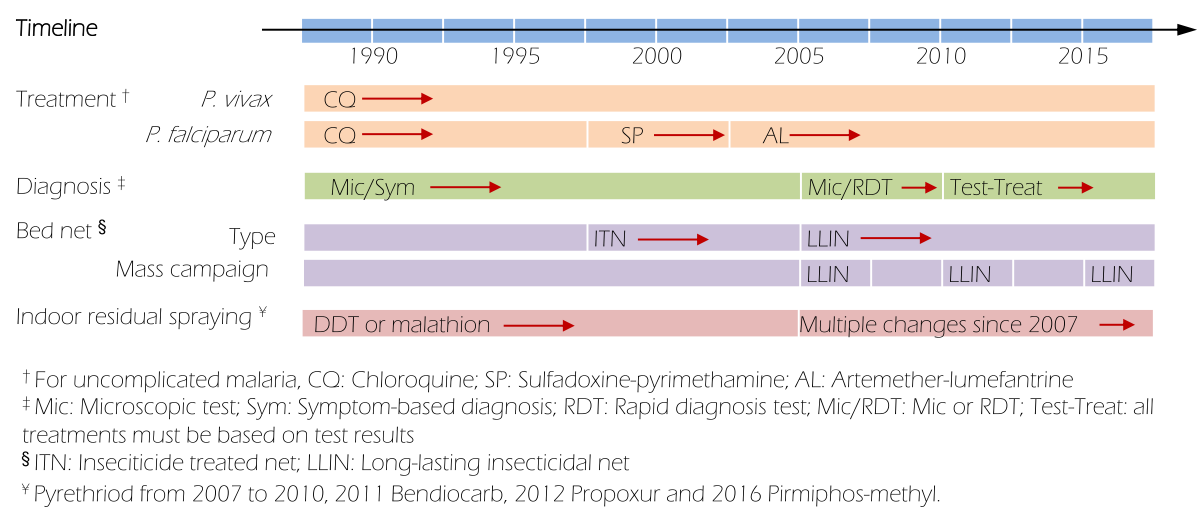

Fig. 1 Malaria intervention scheme in Ethiopia from 1990 to 2016 


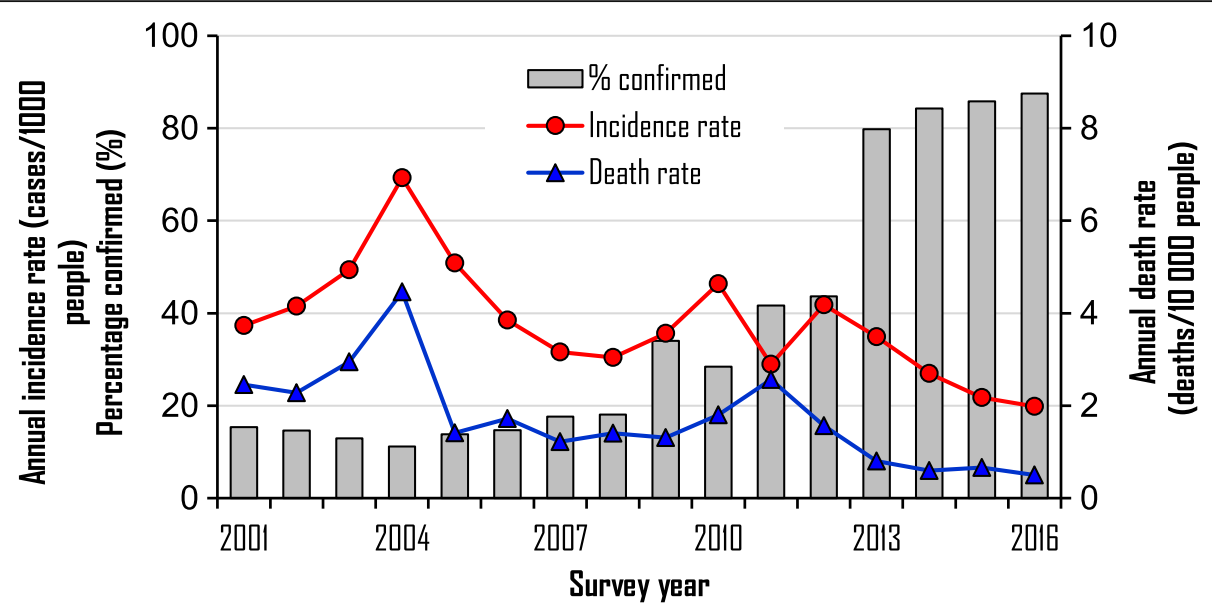

Fig. 2 National level annual total reported malaria cases, deaths due to malaria, and proportion of cases confirmed by microscopy or RDT from 2001 to 2016

2010, and 2012 (Fig. 2). Malaria incidence showed a general declining trend from 2001 to 2016 with some annual variations (Fig. 2). The average annual incidence declined from 43.1 cases per 1000 people annually between 2001 and 2010 (before LLINs were made available free to all malaria affected people) to 29.0 cases per 1000 people between 2011 and 2016. This declining trend was more pronounced from 2014 to 2016 (after two rounds of mass LLIN distribution) when the average annual incidence was 22.9 cases per 1000 people (Fig. 2). Malaria

Table 1 Changes in malaria epidemiological indicators in Ethiopia from 2013 to 2016

\begin{tabular}{llll}
\hline Year & 2013 & 2016 & Change (\%) \\
\hline Total reported cases & & & \\
& 3316013 & 1962996 & -40.8 \\
$\quad$ Confirmed & 2645454 & 1718504 & -35.0 \\
(\% confirmed) & 79.8 & 87.5 & 9.7 \\
$\begin{array}{l}\text { Incidence rate (cases/1000 } \\
\text { population) }\end{array}$ & 36.6 & 20.2 & -44.8 \\
$\begin{array}{l}\text { P. falciparum proportion (\%) } \\
\text { Age }\end{array}$ & 63.8 & 66.5 & 4.2 \\
$\quad<5$ years & & & \\
(\% of total) & 785895 & 319795 & -59.3 \\
$\quad \geq 5$ years & 23.7 & 17.1 & -27.8 \\
Malaria admission & 2530118 & 1553231 & -38.6 \\
$\quad<5$ years & & & \\
(\% of total) & 6602 & 6766 & 2.5 \\
$\quad \geq 5$ years & 16.5 & 16.9 & 2.5 \\
Malaria deaths & 33525 & 33345 & -0.5 \\
$<5$ years & & & -72.1 \\
(\% of total) & 421 & 12 & -58.2 \\
$\quad \geq 5$ years & 5.6 & 2.4 & -30.9 \\
\hline
\end{tabular}

death incidence showed a similar declining trend (Fig. 2). There were 2.1 deaths per 100000 people annually between 2001 and 2010 and 1.1 cases per 1000000 people annually between 2011 and 2016, a drop of $47 \%$. The declining trend was more pronounced from 2014 to 2016, with an annual incidence of 0.6 cases per 100000 people (Fig. 2, Table 1).

\section{Malaria diagnosis and parasite species}

The number of RDT and microscopy tested suspected malaria cases increased significantly from below one million before 2008 to 8.6 million in 2014 and an average of 7.1 million from 2014 to 2016 (Fig. 3). Case confirmation rate by microscopy and/or RDT increased from 79.8\% in 2013 to $87.5 \%$ in 2016 (Table 1). Although there was year-to-year variation, malaria species changed little over time. Plasmodium falciparum accounted for $\sim 60 \%$ of cases (range $55-$ 69\%) and P. vivax 40\% (range 31-45\%) from 2001 to 2016 (Fig. 3, Table 1).

\section{Intensification of malaria interventions and increase in number of health facilities}

FMoH has distributed 18.2 million ITN/LLINs between 2006 and 2007, 13.0 million LLINs in 2010 and 42.4 million LLINs between 2014 and 2016 (Fig. 4a). However, the nation-wide LLIN ownership (proportion of households with at least one LLIN) has been relatively low, $65 \%$ in $2007,55 \%$ in 2011 and $64 \%$ in 2015. IRS coverage increased from $20.0 \%$ in 2007 to $92.5 \%$ in 2016 in epidemic-prone areas located at altitudes $\geq 2500 \mathrm{~m}$ (Fig. 4b). IRS was not implemented in other non-targeted areas. Meanwhile, the number of health facilities nationally has steadily increased from 3612 in 2000 to 6604 in 2005, just before the scale-up of malaria interventions. The total number of health facilities in 2016 was 20283 (Fig. 4c). 


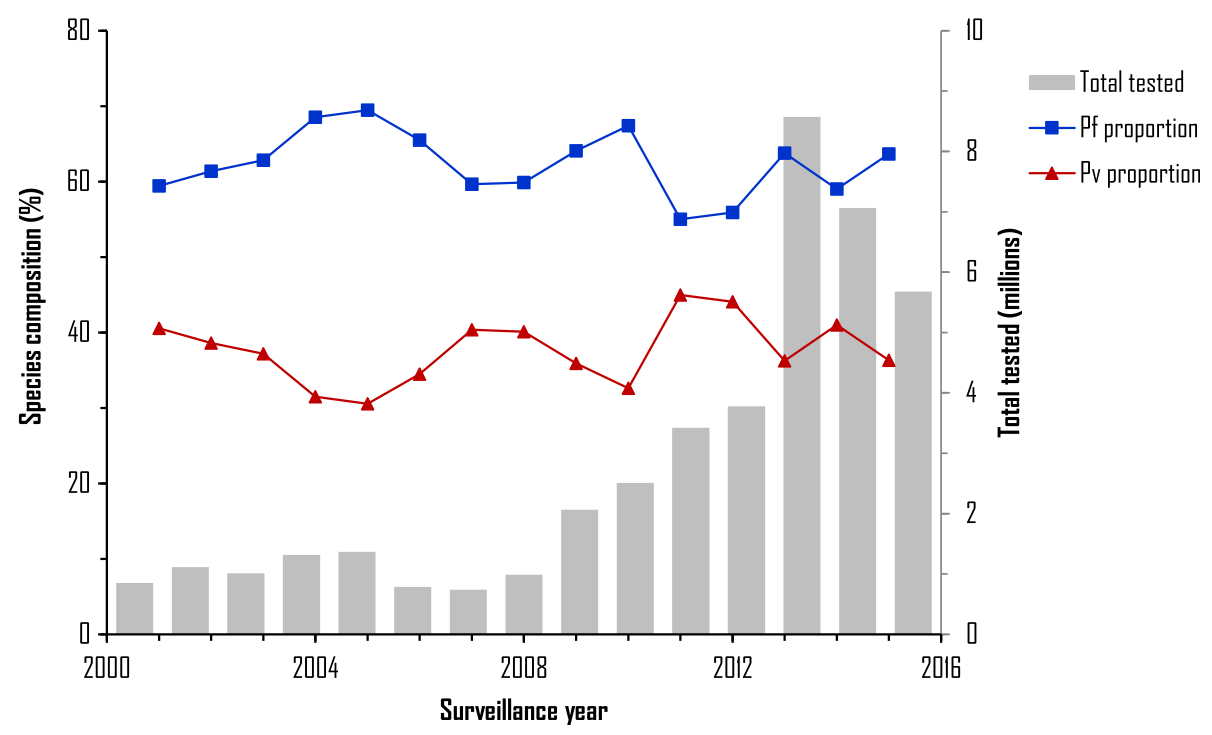

Fig. 3 National level annual total number of suspected cases tested and parasite species composition from 2001 to 2016. Pf: Plasmodium falciparum; Pv: Plasmodium vivax

\section{Changes in spatial transmission patterns}

Overall malaria-related hospital admissions showed no significant change between 2013 and 2016 (Table 1). However, malaria deaths decreased significantly from 2013 to 2016. Reported malaria cases in children $<5$ years decreased by 59.3\% between 2013 and 2016, from a total of 785895 to 319795 , respectively (Table 1). Malaria deaths dropped by $72.1 \%$ over the same time interval, only 12 deaths were reported in 2016 (Table 1).

Spatially, areas of moderate to high transmission areas decreased between 2013 and 2016 (Fig. 5a and b). Areas with API $>100$ (high transmission) significantly shrank whereas areas with API $\leq 5$ (low transmission) significantly increased, especially in the central west region of Ethiopia where high transmission totally disappeared (Fig. 5, Table 2). Overall, the number of high transmission districts dropped from 14.3 to $6.4 \%$ whereas low transmission districts increased from 22.5 to $37.4 \%\left(\chi^{2}=59.97\right.$, degree of freedom $=3, P<0.0001$ ) (Table 2). Proportion of the population in high risk areas decreased from $9.5 \%$ in 2013 to $3.3 \%$ in 2016 while the proportion in low risk areas increased from 26.7 to $42.7 \%\left(\chi^{2}=3.84, d f=3\right.$, $P=0.0501$ ) (Table 2).

The reduction in clinical malaria was not uniform across the country (Fig. 5c). The majority of districts experienced reductions in incidence rates from 2013 to 2016 (Fig. 5c), with two districts having reductions of API $>500$, i.e., reduction in API of 781 in Masha Woreda, Sheka Zone of SNNPR region and 641 in Fagta Lakoma Woreda, Awi Zone of Amhara Region. By contrast, there were 59 districts with noticeable increases in clinical malaria incidence (API > 5) from 2013 to 2016, and two districts with increases in API > 100, i.e., 131 in Awash Fentale Woreda, Zone 3 of Afar Region, and 118 in Itang Woreda, Agnuak Zone of Gambella Region.

\section{Progress has been made and major challenges Progress has been made and future new interventions}

Ethiopia is one of the few countries in sub-Sahara Africa with a policy to provide the main malaria prevention and control services free of charge, including malaria diagnosis and treatment, mosquito nets and IRS [3, 11]. This policy has ensured accessibility of the poor to malaria interventions and, hence, protection from malaria to increase household economic productivity. We emphasize that, in Ethiopia, the success of these policies largely depended on donor funds [1,25-30]. The overall reduction of malaria burden is evident since 2013, reflected in the shrinkage of the malaria map between 2013 and 2016, particularly in the central highlands of Ethiopia where no high transmission was observed in 2016. Anti-relapse therapy with primaquine for $P$. vivax malaria is not currently recommended in malaria-endemic areas [1]. However, the national malaria case management guidelines outline the specific recommendations, including the use of a full 14-day course of primaquine for radical cure of $P$. vivax at malaria elimination-designated districts and single dose primaquine for gametocytocidal activity against P. falciparum in all areas of the country $[26,30]$. With this new intervention, the case burden is expected to decrease in the near future.

There was clear decrease in malaria incidence rate and malaria death rate in Ethiopia from 2001 to 2016; however, malaria case number and incidence remained high -19.8 

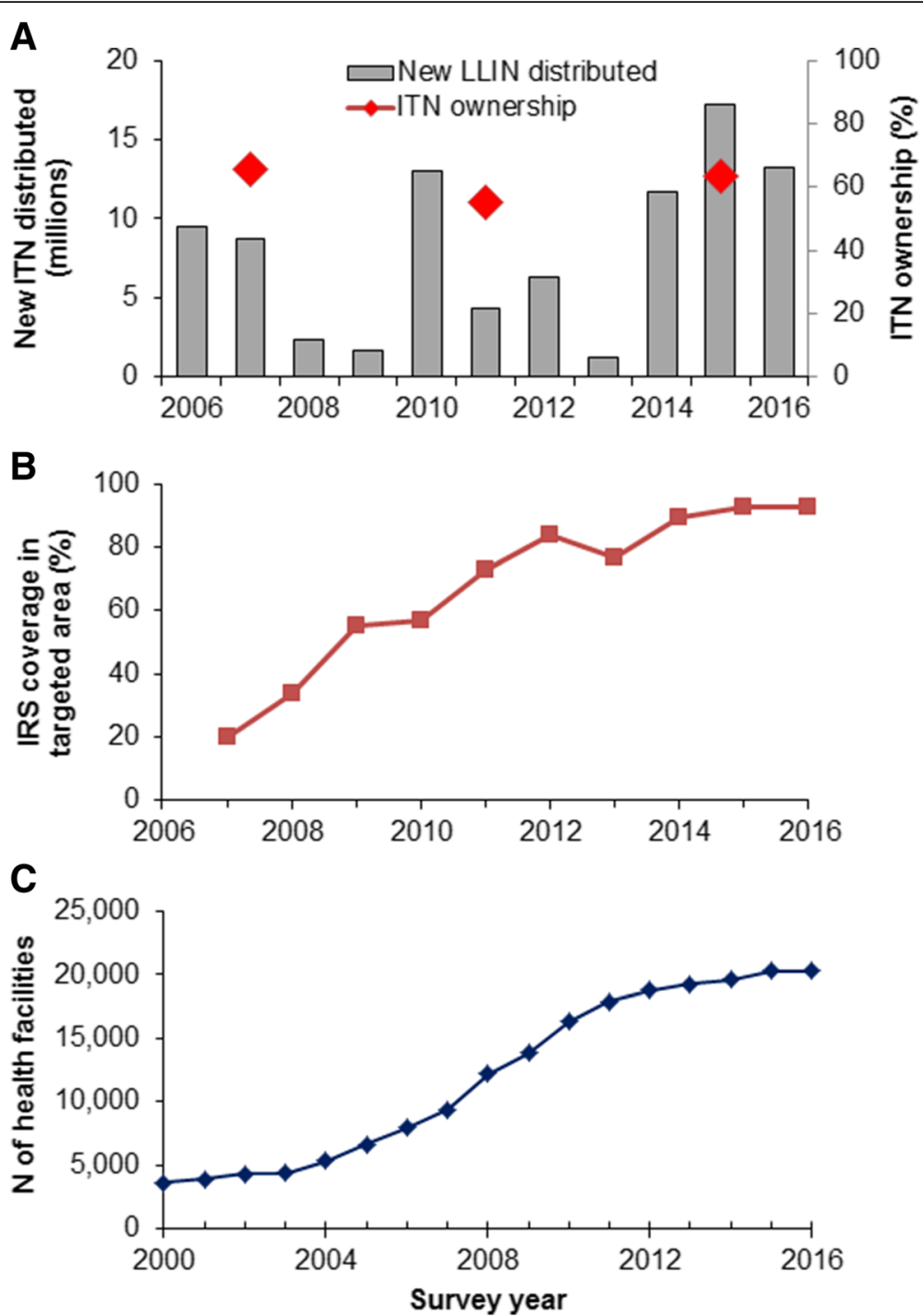

Fig. 4 National level: a. Annual total number of new LLIN distributed from 2006 to 2016 and household LLIN ownership (\% of households with at least one LLIN) in 2007, 2011 and 2015; b. IRS coverage (\%) in targeted areas, and c. Total number of health facilities in Ethiopia from 2000 to 2016. Pf: Plasmodium falciparum; PV: Plasmodium vivax; LLIN: long-lasting insecticidal net; and IRS: indoor residual spraying

cases per 1000 people reported in 2016 exceed the WHO standard for "pre-elimination." Overall LLIN ownership and IRS coverage also continue to be low. In 2016, LLIN ownership rate was $62 \%$ nation-wide, which is far lower than its neighbouring country of Kenya [32, 33]. IRS coverage was even lower, nation-wide average coverage of $23 \%$ from 2014 to 2016 [32]. Therefore, there is ample opportunity to increase LLIN and IRS coverage which would be expected to decrease malaria transmission further. There is a clear need to improve the mechanism of LLIN distribution so that all at risk populations can be adequately covered.

\section{New risk factors}

Although the FMoH of Ethiopia has classified areas at altitudes $>2000 \mathrm{~m}$ as malaria-free zones [10], they are not entirely malaria free at the district level. In fact, many of these districts have moderate APIs according to this study. These areas may have scattered seasonal micro-geographic local transmissions due to local environmental suitability $[10,34]$. For example, construction of dams might increase malaria transmission in the valleys of highland areas where overall malaria transmission was very low [35]. These dams create transmission hot spots along valleys where irrigation is important for farming [36]. Population migration and 


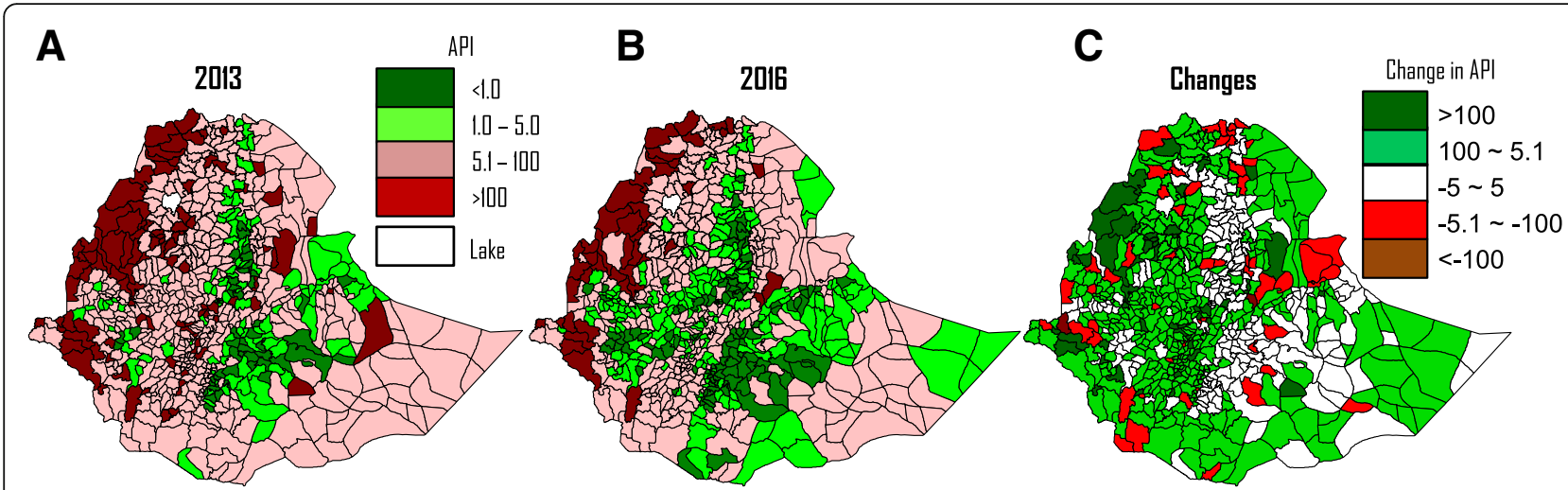

Fig. 5 Malaria transmission intensity maps by districts in a: 2013, b): 2016, and c): Difference in API between 2013 and 2016 (positive indicating decrease and negative indicating increase). APl: annual parasite incidence per 1000 population

treatment-seeking behaviour may have contributed to the high level of API observed in these highlands [37]. Further investigation of this issue is warranted.

\section{Confirmed cases are still high}

There have been significant advances in malaria diagnosis and prevention in Ethiopia since the beginning of scale up in $2004[8,28,38,39]$. Before 2008 , over $80 \%$ of the reported cases were presumptive cases lacking parasitological confirmation. Presumed treatment has declined greatly in the last few years. For example, in 2016, the annual performance report showed that 6367309 suspected cases were examined by microscopy or RDTs, with a total number of 1718504 confirmed cases by either method. Meanwhile, the FMoH reported the total number of clinical malaria cases to be 1962996 , and the proportion of clinically diagnosed cases based on symptoms alone reduced to $12.5 \%$ in 2016 . This was a result of successful implementation of the malaria test-treat policy.

\section{Challenges for further reducing malaria transmission}

Malaria transmission is very heterogeneous across Ethiopia. The malaria burden is still high in western Ethiopia near the borders of Sudan and South Sudan. This suggests that these high burden areas need to be prioritized to sustain the gains made so far and achieve malaria elimination. In central Ethiopia, particularly the central highlands, malaria transmission has been significantly reduced, and many of these areas have reached pre-elimination levels of transmission. These results are similar to those of a previous study showing a reduction in malaria incidence in central Ethiopia after ITN distribution [40-42]. Nevertheless, malaria epidemiology in western Ethiopia has clearly been under-studied [10, 38, 39]. In addition, dealing with how to prevent resurgence and re-introduction is a new challenge for the central highlands. Finally, in the Somalia Region of eastern Ethiopia bordering Somalia where malaria reporting is less accurate due to inadequate capacity, better surveillance systems are desperately needed in the era of malaria elimination [10].

\section{Conclusions}

Malaria morbidity and mortality have significantly declined in Ethiopia since 2000. However, transmission remains high in the western border area near Sudan and South Sudan. Appropriate vector control methods that target outdoor transmission are needed, especially for migrant workers and for people who work at night. Additional efforts are needed to target the high transmission area of western Ethiopia to sustain achievements made to date and hasten the endeavour of achieving malaria elimination.

Table 2 Changes in distribution of malaria transmission intensity and affected populations at district level in Ethiopia from 2013 to 2016

\begin{tabular}{|c|c|c|c|c|c|c|c|c|c|c|}
\hline \multirow[t]{2}{*}{$\overline{\mathrm{API}}$} & \multicolumn{4}{|c|}{ Number of districts } & \multirow[t]{2}{*}{ Change in percentage $^{a}$} & \multicolumn{4}{|c|}{ Total population (\%) } & \multirow[t]{2}{*}{ Change in percentage $^{a}$} \\
\hline & 2013 & $\%$ & 2016 & $\%$ & & 2013 & $\%$ & 2016 & $\%$ & \\
\hline$<1.0$ & 60 & 7.2 & 92 & 10.9 & 3.7 & 7887232 & 9.2 & 11730861 & 12.7 & 3.4 \\
\hline $1.0-5.0$ & 128 & 15.3 & 224 & 26.5 & 11.2 & 14941965 & 17.5 & 27787000 & 30.0 & 12.5 \\
\hline $5.1-100$ & 527 & 63.2 & 475 & 56.2 & $(7.0)$ & 54487279 & 63.8 & 50005687 & 54.0 & $(9.8)$ \\
\hline$>100$ & 119 & 14.3 & 54 & 6.4 & $(7.9)$ & 8133374 & 9.5 & 3093995 & 3.3 & $(6.2)$ \\
\hline Total & 834 & & 845 & & & 85449850 & & 92617543 & & \\
\hline
\end{tabular}

${ }^{a}$ Difference in percentages from 2013 to 2016, numbers in brackets indicating negative 


\section{Additional file}

Additional file 1: Multilingual abstracts in the five official working languages of the United Nations. (PDF $423 \mathrm{~kb}$ )

\section{Abbreviations}

ACT: Artemisinin-based combination therapy; AL: Artemether-lumefantrine; API: Annual parasite incidence; CQ: Chloroquine; FMoH: Federal Ministry of Health; GFATM: Global Fund to Fight AIDS, Tuberculosis and Malaria; HEW: Health Extension Workers; HMIS: Health Management Information System; IRS: Indoor residual spraying; ITN: Insecticide-treated net; LLIN: Longlasting insecticidal net; Pf: Plasmodium falciparum; PMI: President's Malaria Initiative; PV: Plasmodium vivax; RDT: Rapid diagnosis test; SNNPR: Southern Nations, Nationalities, and Peoples' Region, Ethiopia; SP: Sulfadoxinepyrimethamine; WHO: World Health Organization

\section{Acknowledgments}

We would like to thank staffs at the Federal Ministry of Health of Ethiopia for organizing the data, and staffs of the University of California, Irvine, California, USA for supporting the data analysis and review.

\section{Funding}

This study was supported by grants from the National Institutes of Health (D43 TW001505, R01 A1050243 and U19 Al129326 to G. Yan).

\section{Availability of data and materials}

All data generated or analysed during this study and supporting the conclusions of this article are included within the article.

\section{Authors' contribution}

GY, GZ and ESH conceived and designed the study concept. ESH, GT and GZ collected the data. GZ and MCL conducted data analysis. ESH, GZ, CK, EHS and JK wrote the paper. All authors reviewed the paper and approved the final version.

\section{Ethics approval and consent to participate}

Not applicable

\section{Consent for publication}

Not applicable.

\section{Competing interests}

The authors declare that they have no competing interests.

\section{Author details}

${ }^{1}$ National Malaria Program, Federal Ministry of Health, Addis Ababa, Ethiopia. ${ }^{2}$ Program in Public Health, University of California, Irvine, CA 92697, USA. ${ }^{3}$ Center for Global Health and Diseases, Case Western Reserve University, Cleveland, OH, USA.

Received: 3 May 2018 Accepted: 27 September 2018

\section{Published online: 05 November 2018}

\section{References}

1. World Health Organization. World Malaria Report 2017. http://www.who. int/malaria/publications/world-malaria-report-2016/report/en/. Accessed 30 Mar 2018

2. McCann JC. The historical ecology of malaria in Ethiopia. Deposing the spirits. Athens: Ohio University Press; 2014.

3. Ministry of Health. National strategic plan for malaria prevention, control and elimination in Ethiopia, 2011-2015. Addis Ababa: Ministry of Health of Ethiopia; 2010. http://www.nationalplanningcycles.org/sites/default/files/ country_docs/Ethiopia/ethiopia_malaria_national_strategic_plan_20112015_130810.pdf. Accessed 30 Mar 2018

4. Yalew WG, Pal S, Bansil P, Dabbs R, Tetteh K, Guinovart C, et al. Current and cumulative malaria infections in a setting embarking on elimination: Amhara, Ethiopia. Malar J. 2017:16:242

5. Delil RK, Dileba TK, Habtu YA, Gone TF, Janfa T. Magnitude of malaria and factors among febrile cases in low transmission areas of Hadiya zone, Ethiopia: a facility based cross sectional study. PLoS One. 2016, e0154277;11.
6. Zhou G, Yewhalaw D, Lo E, Zhong D, Wang X, Degefa T, et al. Analysis of asymptomatic and clinical malaria in urban and suburban settings of southwestern Ethiopia in the context of sustaining malaria control and approaching elimination. Malar J. 2016;15:250.

7. Aregawi M, Lynch M, Bekele W, Kebede H, Jima D, Taffese HS, Yenehun MA, Lilay A, Williams R, Thomson M, Nafo-Traore F, Admasu K, Gebreyesus TA, Coosemans M. Time series analysis of trends in malaria cases and deaths at hospitals and the effect of antimalarial interventions, 2001-2011, Ethiopia. PLoS One. 2014, e106359;9.

8. Gebre B, Negash Y. Severe malaria among children in Gambella, western Ethiopia. Ethiop J Health Dev. 2002;16:61-70.

9. PMI. Africa IRS (AIRS) Project Indoor Residual Spraying (IRS 2) Task Order Six. Ethiopia 2016 End of spray report. Bethesda, MD: Abt Associates Inc; 2016.

10. Ethiopian Public Health Institute. Ethiopia National Malaria Indicator Survey 2015. Addis Ababa: Ethiopian Public Health Institute; 2016. https://www. ephi.gov.et/images/pictures/download2009/MIS-2015-Final-ReportDecember-_2016.pdf. Accessed 30 June 2017

11. Ministry of Health. National Malaria Guidelines. 3rd ed. Addis Ababa: Federal Ministry of Health of Ethiopia; 2012. https://www.medbox.org/nationalmalaria-guidelines-ethiopia/download.pdf. Accessed 30 Mar 2018

12. Lo E, Hemming-Schroeder E, Yewhalaw D, Nguyen J, Kebede E, Zemene E, et al. Transmission dynamics of co-endemic Plasmodium vivax and $P$ falciparumin Ethiopia and prevalence of antimalarial resistant genotypes. PLoS Negl Trop Dis. 2017;11:e0005806.

13. Gething PW, Elyazar IQF, Moyes Cl, Smith DL, Battle CE, Guerra CA, et al. A long neglected world malaria map: Plasmodium vivax endemicity in 2010. PLoS Negl Trop. 2012;6:e1814.

14. Teka H, Petros B, Yamuah L, Tesfaye G, Elhassan I, Muchohi S, et al. Chloroquine-resistant Plasmodium vivax malaria in Debre Zeit, Ethiopia. Malar J. 2008;7:220.

15. Assefa M, Eshetu T, Biruksew A. Therapeutic efficacy of chloroquine for the treatment of Plasmodium vivax malaria among outpatients at Hossana health care Centre, southern Ethiopia. Malar J. 2015;14:458.

16. Ketema T, Bacha K, Birhanu T, Petros B. Chloroquine-resistant Plasmodium vivax malaria in Serbo town, Jimma zone, south-West Ethiopia. Malar J. 2009;8:177.

17. Beyene $H B$, Beyene MB, Ebstie YA, Desalegn Z. Efficacy of chloroquine for the treatment of vivax malaria in Northwest Ethiopia. PLOS One. 2016:11:e0161483.

18. World Health Organization. Implementation of indoor residual spraying of insecticides for malaria control in the WHO African region report: WHO regional Office for Africa; 2007.

19. Federal Ministry of Health. Insecticide Treated Nets (ITNs): National Strategic Plan for going to scale with coverage and utilization in Ethiopia, 2004 - 2007. Addis Ababa: Federal Ministry of Health; 2004

20. Alene GD, Bennett S. Chloroquine resistance of Plasmodium falciparum malaria in Ethiopia and Eritrea. Tropical Med Int Health. 1996;1:810-5.

21. Teklehaimanot A. Chloroquine-resistant Plasmodium falciparum malaria in Ethiopia. Lancet. 1986;2:127-9.

22. Jima D, Tesfaye G, Medhin A, Kebede A, Argaw D, Babaniyi O. Efficacy of sulfadoxine-pyrimethamine for the treatment of uncomplicated falciparum malaria in Ethiopia. East Afr Med J. 2005;82:391-5.

23. Kassa M, Sileshi M, Mohammed H, Taye G, Asfaw M. Development of resistance by Plasmodium falciparum to sulfadoxine/pyrimethamine in Amhara region, Northwestern Ethiopia. Ethiop Med J. 2005;43:181-7.

24. Federal Ministry of Health. Malaria diagnosis and treatment guidelines for health Workers in Ethiopia. 2nd ed. Addis Ababa: Federal Ministry of Health; 2004.

25. Federal Ministry of Health. National Malaria Program Monitoring and Evaluation Plan 2014 - 2020. Addis Ababa: Federal Ministry of Health. p. 2014.

26. Federal Ministry of Health. National Strategic Plan for Malaria 2017-2020. Addis Ababa: Federal Ministry of Health; 2017. https://www.medbox.org/ national-malaria-guidelines-ethiopia/download.pdf. Accessed 30 Mar 2018

27. PMI. Ethiopia Malaria Operational Plan FY 2014. Addis Ababa: USAID, Ethiopia; 2014.

28. PMI. Ethiopia Malaria Operational Plan FY 2015. Addis Ababa: USAID, Ethiopia; 2015

29. PMI. Ethiopia Malaria Operational Plan FY 2016. Addis Ababa: USAID, Ethiopia; 2016

30. PMI. Ethiopia Malaria Operatinal Plan FY 2017. Addis Ababa: USAID, Ethiopia; 2017. https://www.pmi.gov/docs/default-source/default-document-library/ malaria-operational-plans/fy17/fy-2017-ethiopia-malaria-operational-plan. pdf?sfvrsn=6. Accessed 5 Nov 2017 
31. National Malaria Control Team, Ethiopian Public Health Institute, World Health Organization, Addis Ababa University and the INFORM Project. An epidemiological profile of malaria in Ethiopia. A report prepared for the Federal Ministry of Health, Ethiopia, the roll Back malaria partnership and the department for international development, UK; 2017.

32. World Health Organization. World malaria report 2017. Geneva: WHO; 2018. http://www.who.int/malaria/publications/world-malaria-report-2017/en/. Accessed 30 Mar 2018

33. Zhou G, Lee MC, Githeko AK, Atieli HE, Yan G. Insecticide-treated net campaign and malaria transmission in western Kenya: 2003-2015. Front Public Health. 2016:4:153.

34. Federal Ministry of Health. National Five-year Strategic Plan for Malaria Prevention \& Control in Ethiopia 2006-2010. Addis Ababa: Federal Ministry of Health; 2006.

35. Sena L, Deressa W, Ali A. Dynamics of Plasmodium falciparium and Plasmodium vivax in a micro-ecological setting, Southwest Ethiopia: effects of altitude and proximity to a dam. BMC Infect Dis. 2014;14:625.

36. Kibret S, Wilson GG, Tekie H, Petros B. Increased malaria transmission around irrigation schemes in Ethiopia and the potential of canal water management for malaria vector control. Malar J. 2014;13:360.

37. Degefa T, Zeynudin A, Godesso A, Michael YH, Eba K, Zemene E, et al. Malaria incidence and assessment of entomological indices among resettled communities in Ethiopia: a longitudinal study. Malar J. 2015;14:24.

38. Jima D, Getachew A, Bilak H, Steketee RW, Emerson PM, Graves PM, et al. The Ethiopia malaria Indicator survey working group. Malaria indicator survey 2007, Ethiopia: coverage and use of major malaria prevention and control interventions. Malar J. 2010;9:58.

39. Federal Ministry of Health. Ethiopia National Malaria Indicator Survey 2011 technical summary. Addis Ababa: Federal Ministry of Health; 2012.

40. Otten M, Aregawi M, Were W, Karema C, Medin A, Bekele W, et al. Initial evidence of reduction of malaria cases and deaths in Rwanda and Ethiopia due to rapid scale-up of malaria prevention and treatment. Malar J. 2009;8:14

41. Toyama Y, Ota M, Molla G, Beyene BB. Sharp decline of malaria cases in the Burie Zuria, Dembia, and Mecha districts, Amhara region, Ethiopia, 20122014: descriptive analysis of surveillance data. Malar J. 2016;15:104

42. Deribew A, Dejene T, Kebede B, Tessema GA, Melaku YA, Misganaw A, et al. Incidence, prevalence and mortality rates of malaria in Ethiopia from 1990 to 2015: analysis of the global burden of diseases 2015. Malar J. 2017;16:271.

Ready to submit your research? Choose BMC and benefit from:

- fast, convenient online submission

- thorough peer review by experienced researchers in your field

- rapid publication on acceptance

- support for research data, including large and complex data types

- gold Open Access which fosters wider collaboration and increased citations

- maximum visibility for your research: over $100 \mathrm{M}$ website views per year

At $\mathrm{BMC}$, research is always in progress.

Learn more biomedcentral.com/submissions 